\title{
Design of Water Quality and Quantity Ecological Compensation Monitoring System based on Wireless Sensor Network
}

\author{
Yizhuo Wang ${ }^{1}$, Rongjin Yang ${ }^{2}$, Lizeyan Yin $^{3}$, Xiuhong Li $^{1+}$,Qiang Liu ${ }^{1}$, Rongzhen Xun ${ }^{1}$, \\ Kaixiang Yang ${ }^{1}$,Xuejie Hao $^{1}$,Shumin Wang ${ }^{1}$ \\ ${ }^{1}$ State Key Laboratory of Remote Sensing Science, College of Global Change and Earth System Science, \\ Beijing Normal University, No.19, Xinjiekou Wai Street, Haidian District, Beijing 100875, China; \\ ${ }^{2}$ Chinese Research Academy of Environmental Sciences, No.8, Da Yang Fang, An Wai, Chao Yang, Beijing \\ 100012, China; \\ ${ }^{3}$ Univ Clermont Auvergne, INP, Institute Supérieur d’Informatique de Modélisation et leurs Applications, \\ ISIMA, Aubière Cedex, France
}

\begin{abstract}
As an effective means to solve the environmental resource problems of the river basin and coordinate the economic and social development of the upstream and downstream, a number of pilot projects have been carried out internationally. The establishment of river basin ecological compensation standards requires monitoring of a number of indicators in the river basin to formulate corresponding assessment targets.In order to solve the problems of single monitoring indicators, inconsistent water quality and hydrological monitoring points, and low monitoring accuracy in the current river basin monitoring system in my country, this paper designs a pollutant flux ecological compensation monitoring system that can be used for ecological compensation in the river basin. Compensated river temperature, redox potential, conductivity, $\mathrm{pH}$, transparency, ammonia nitrogen, permanganate index, water level, flow rate and flow rate and other indicators for real-time monitoring. The system realizes the real-time collection, transmission, display and storage of water environment and water resources data in the river basin, and meets the current needs of constructing water quality and quantity river basin ecological compensation with the advantages of low cost, flexible and convenient layout, and high precision.
\end{abstract}

Keywords: river basin ecological compensation, wireless sensor network, flux monitoring, ecological environment monitoring.

\section{Introduction}

Since the 1990s, with the continuous improvement of countries' awareness of the value of watershed ecological services, with the funding of international organizations represented by IFAD and CIIFAD, a batch of pilot projects for PWES(Payment for Watershed Ecological Service) in various developing countries have been implemented[1].These PWES projects have their respective focuses: the PSA(Pagopor Servicios Ambientales) project in Costa Rica in Central and South America is the first project to explore payment for environmental services of the river basin ecosystem and has successfully carried out long-term operations[2].The Working for Water Program project in South Africa, Africa, has become a model for coordination of fairness, efficiency and sustainability in similar projects in African countries by giving economic value to ecosystem services and eliminating invasive alien plants[3].PWES projects carried out in various modes abroad have diverse sources of funds, but the size of the funds needs to be determined by the value of the ecosystem services that the river basin can provide, such as clean and abundant water resources, functions of conserving water sources and maintaining soil and water, biodiversity, etc. How to effectively quantify the value of ecological services requires a special monitoring system to monitor and evaluate the various ecological environmental indicators of the river, and use this as a basis for the calculation of ecological compensation.

In China, Liaoning, Zhejiang, Hebei, Shanxi, Jiangsu, Guangdong, Hubei, Jiangxi and other provinces

\footnotetext{
${ }^{+}$Corresponding author. Tel.: +86 13621166693; fax: None.

E-mail address: lixh@bnu.edu.cn.
} 
have successively launched a series of watershed ecological compensation pilot projects, mainly for crossborder water quality ecological compensation [4]. The water quality of most pilot basins and cross-border sections has improved significantly, and the pilot has achieved certain results. However, due to the lack of watershed design and watershed perspective of the transboundary ecological compensation in the river basin, there is no unified plan for ecological compensation: the monitoring of water quality, water quantity, and water ecological indicators involved in ecological compensation is not in place, and it is difficult to quantify the value of ecosystem services. The scale of ecological compensation funds lacks basis and is highly dependent on the central finance. A stable and long-term co-construction, sharing and win-win mechanism has not yet been established [5]

Some provinces and cities in my country have made some attempts to construct an ecological compensation monitoring system, but their monitoring system is mainly assessed based on water quality monitoring data [6-8], ignoring the monitoring of water volume data, resulting in unclear calculations of the total amount of pollutants. The assessment data is not scientific and comprehensive enough to fully reflect the objective conditions of river and lake water pollution. The currently adopted ecological compensation monitoring system uses a single target of monitoring data, which leads to one-sided assessment. Specifically, the river basin ecological compensation mechanism with water quality as the target is evaluated according to the annual average pollutant concentration of the cross-border section, and it cannot fully and effectively reflect the impact of changes in the water volume during the flood and non-flood periods of the year. At the same time, the regional differences and demand of water resources in different river basins also show that it is difficult to implement compensation in place only considering water quality standards. At present, how to define the weight of water quality compensation based on the distribution of water resources in the upstream and downstream of the river basin, downstream development needs and the ecological protection and construction work carried out by the upstream is still one of the factors that needs to be considered in many aspects. The water volume of the rivers in the north is the most important indicator, and it cannot be assessed only by the cross-sectional monitoring data. In addition, the departments responsible for monitoring data are different, and data is not shared, resulting in incomplete data. At present, the environmental protection department is responsible for the deployment and data collection of domestic water quality monitoring. Water quantity data is deployed and collected by the water affairs department. And the monitoring points are sometimes not uniform, so the matching of the data is not high.

Taking the above requirements into consideration, this paper designs a flux ecological compensation monitoring system based on a wireless sensor network. The goal is to build a low-cost, reusable, and convenient to change location (not to be installed in the assessment section, but also in other areas of the basin. Flexible deployment) to meet the needs of building a comprehensive ecological compensation.

\section{Study Area}

In this paper, the largest branch of the Haihe River in northern China-the Yongding River Basin is used as the research area. The Yongding River is about $747 \mathrm{~km}$, with a vast drainage area of up to $47016 \mathrm{~km}^{2}$ [9]. Its upstream flows through China's water-deficient provinces-Inner Mongolia, Shanxi, and Hebei, and its downstream flows through the Chinese capital Beijing, and the municipality of Tianjin into the Bohai Sea. As the mother river of the capital Beijing, Yongding River is the main source of groundwater resources in western Beijing [10].With the accelerated development of urbanization of cities and towns along the Yongding River, problems such as water scarcity, water environmental pollution, and degradation of water ecological functions have become increasingly apparent [11].Especially due to the over-exploitation of water resources in the upstream and the vigorous construction of water conservancy facilities to intercept river water, and the impact of sewage discharge from power plants, coal mines, and farmland irrigation along the line, the water quality of the Yongding River Basin has deteriorated and the eutrophication trend is obvious [12].The pollution and resource problems in the Yongding River Basin have attracted the attention of the National Committee of the Chinese People's Political Consultative Conference, the Ministry of Ecology and Environment, the Ministry of Water Resources, and the four provincial governments, etc., in order to maintain and realize the sustainable use of water resources and a clean water environment in the Yongding River Basin. Safety, better realization of the goal of coordinating economic development and environmental 
protection, and speeding up the construction of a comprehensive, accurate and efficient monitoring system are the necessary prerequisites for the establishment of a sound river basin ecological compensation mechanism.

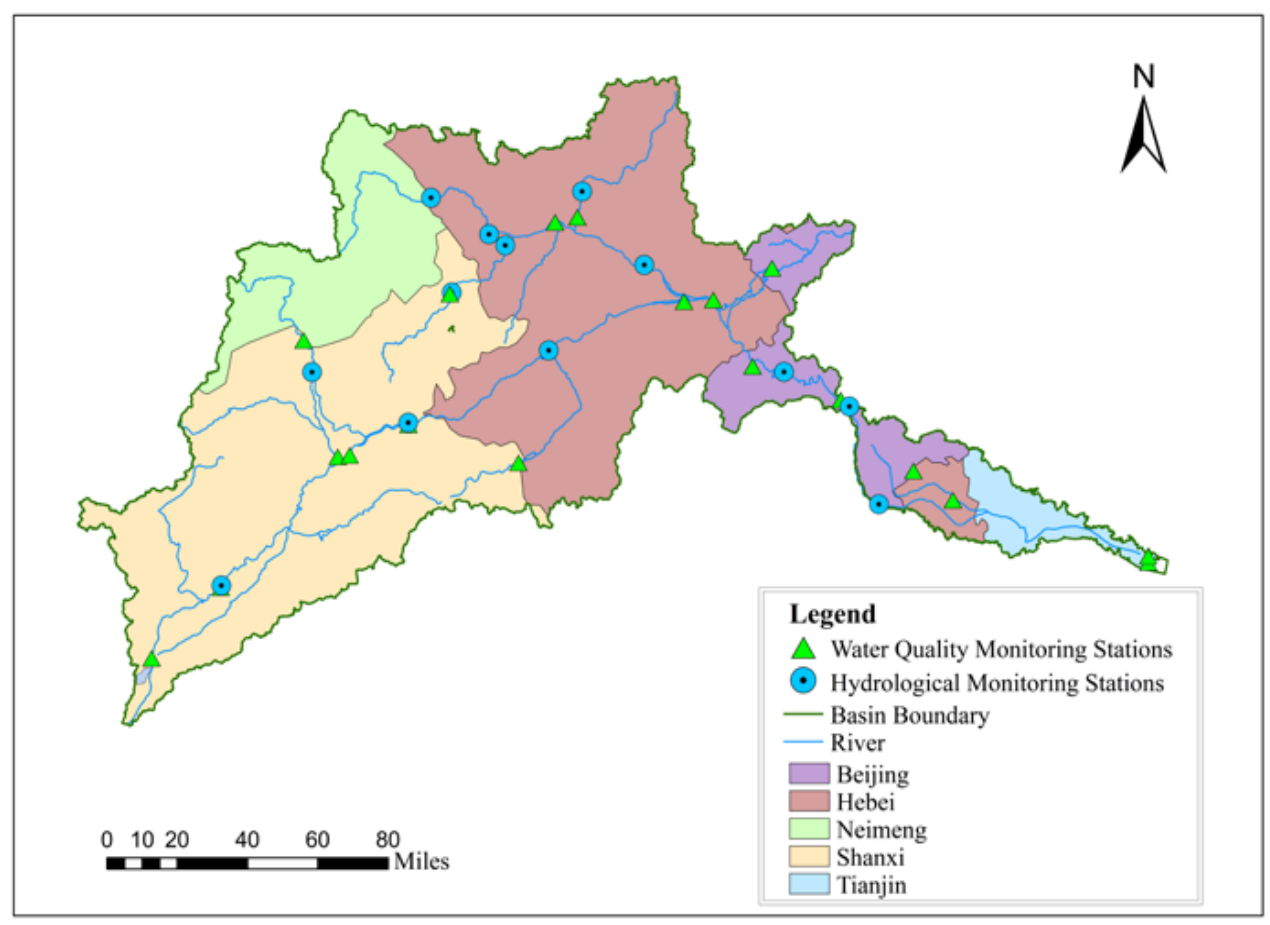

Fig. 1: Overview of the study area and monitoring points

At present, 17 water quality monitoring sections and 13 hydrological monitoring stations have been deployed in the whole basin (Fig 1). Except for the automatic monitoring of state-controlled water quality sections, other water quality monitoring stations require sampling once a month, 12 times a year. The water quality monitoring section has low monitoring frequency and low spatial distribution density. There are differences in the locations of hydrological sites and water quality sites. The water quality and hydrological sites are not uniform, and data is difficult to match. Therefore, in order to carry out the pilot ecological compensation of the flux in the Yongding River Basin, wireless sensor network technology can be used to construct a high-precision, low-cost, convenient and flexible ecological compensation monitoring system.

\section{System Design}

\subsection{Overall System Framework}

The high-temporal precision flux ecological compensation monitoring system can realize the monitoring of water quality and water volume, and reserves the interface to ensure the access of more parameters in the later period. The parameters that can be monitored include temperature, water level, dissolved oxygen, redox potential, conductivity, $\mathrm{pH}$, transparency, flow rate, ammonia nitrogen, permanganate index, and flow rate. A large number of layouts can be realized through low-cost design. Through flexible setting of acquisition frequency, monitoring with high time accuracy and high spatial accuracy can be achieved, making up for the lack of existing monitoring points, and data is not shared between different departments, and the standards Problems caused by unification.

The system structure diagram of the designed flux ecological compensation monitoring system (Hereinafter referred to as system) is shown in Fig. 2 below: 


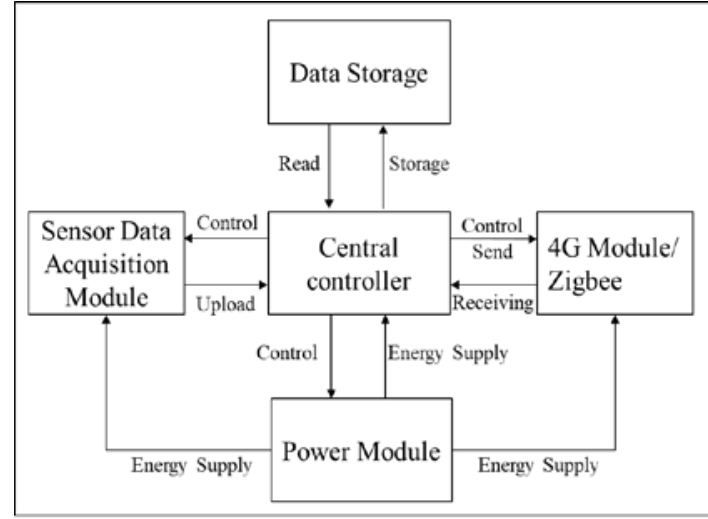

Device Side

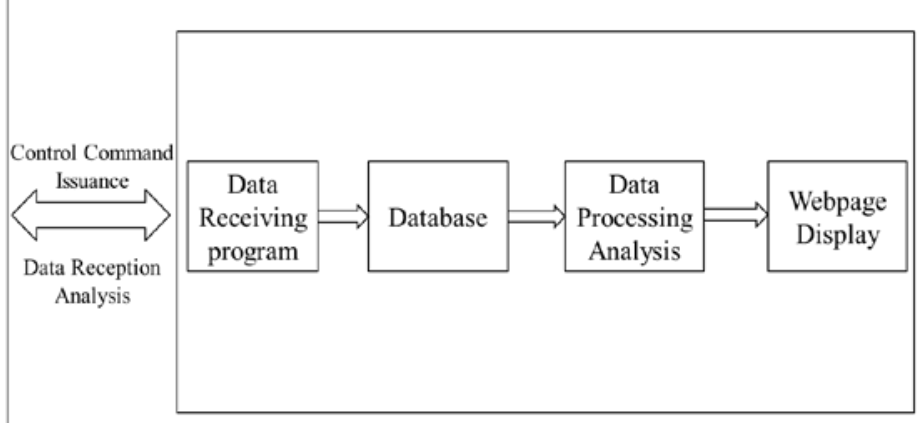

Server Side

Fig. 2: System structure diagram

The system consists of 5 modules: sensor data acquisition module, data storage module, power supply module, central controller module, communication module and big data center.

The sensor data acquisition module mainly includes two parts: the sensor and the data transmission. The core motherboard of the device can control the working time of the sensor and transmit the data to the motherboard through the RS232 serial port USART protocol. The motherboard then stores the data in the local SD card and sends it remotely.

The function of the autonomous management module is to complete the scheduling of requested tasks, remote code upgrades, equipment failure detection and remote execution of monitoring tasks under unmanned maintenance.

The communication module includes multiple interfaces (serial port, 485 bus interfaces, etc.) of the collection and transmission module, energy level estimation, dynamic scheduling of communication tasks through energy-saving strategies, and realization of communication task aggregation and unified services to reduce communication energy consumption. There is also a separate set of ZIGBEE modules, which are used to receive remote control command information and the monitoring position of the mobile system.

The power management module coordinates solar energy acquisition and energy consumption control of hardware devices through the energy management control strategy library. Based on the current task operation, the energy consumption is dynamically controlled to minimize energy consumption.

The special waterproof and thermal insulation package are for the normal operation of the equipment in the special environment of water or low temperature.

\subsection{Central Controller Module}

The core processor is the core part of the entire device. It controls the operation of the entire system, including important tasks such as time management, task deployment, data acquisition, storage and communication, and power management. Its composition mainly includes: microprocessor, peripheral circuit, clock management.

In order to design the system to meet the requirements of low power consumption, ST's 32-bit ARMSTM32F107 was selected as the main control chip. This chip belongs to the Cortex ${ }^{\circledR}$-M3 core, with a clock frequency of up to $72 \mathrm{MHz}$ and a maximum operating current of only $68.4 \mathrm{~mA}$, which meets the requirements of system operating speed and low power consumption. The chip has three low-power modes: sleep mode, the maximum current is $49 \mathrm{~mA}$ in the mode, only the chip CPU stops running but all its peripherals continue to run, once an interrupt or event occurs, the system will wake up the CPU and enter the normal working state. The stop mode can reach the minimum power consumption of $1.3 \mathrm{~mA}$ of the processor while keeping the contents of SRAM and registers not falling. All the internal clocks stop running at $1.8 \mathrm{~V}$ and the PLL, internal clock and external clock are disabled. This mode can be excited by an external signal interrupt or event. The standby mode can achieve the lowest power consumption of the processor, with a typical current of only 3.8uA. In this mode, all internal 1.8V circuits are turned off. Except for the backup 
registers and standby circuits, the contents of SRAM and other registers are lost. To wake up this mode, you need to reset on the WKUP pin or use the RTC alarm to wake up.

The peripheral circuit mainly includes a reset circuit and a JTAG circuit, mainly for the software program burning and resetting of the chip.

In order to ensure the normal operation of the entire system under low temperature conditions and a more accurate time system for the processor, this system uses an external $12 \mathrm{MHz}$ temperature-compensated crystal oscillator to provide the chip with a basic clock source.

\subsection{Sensor Data Acquisition Module}

\subsubsection{Index Selection}

For the selection of related indicators of water environment quality in the basin, according to the "Surface Water Environmental Quality Standards" (GB3838-2002) applicable to rivers, lakes, canals, channels, reservoirs and other surface water areas in the People's Republic of China, the system indicators should include at least $\mathrm{pH}$ value, Dissolved oxygen, permanganate index, ammonia nitrogen. In addition, the data released by the Bureau of Ecology and Environment showed that the monitoring sections also monitored physical indicators such as temperature, redox potential, electrical conductivity, and transparency. The monitoring indicators for the amount of water resources in the river basin will cover the monitoring indicators of the hydrological stations in each region, such as water level, flow, and flow velocity. Therefore, the observation capabilities of the design node should include temperature, water level, dissolved oxygen, redox potential, conductivity, $\mathrm{pH}$, transparency, flow rate, ammonia nitrogen, permanganate index and flow parameters, which can also be appropriately added according to the environmental conditions of different basins. Other monitoring indicators, and provide additional interfaces for the access of its sensors.

\subsubsection{Data Collection}

This part includes temperature, water level, dissolved oxygen, redox potential, conductivity, $\mathrm{pH}$, transparency, flow rate, ammonia nitrogen, permanganate index and flow parameter sensors. The sensors communicate or interact with the main board through the data acquisition board. For different types of sensors, the acquisition board interacts with the main board in different ways: for digital sensors (such as water level sensors), the acquisition board only provides an interface that allows the sensor to communicate with the motherboard; for analog sensors (such as temperature Sensors, conductivity sensors, etc.), the electrical signal output by the sensor is amplified and converted by the acquisition board and then passed to the main board for processing through a dedicated interface. An external sensor can also be connected through the RS232 interface, and the acquisition board is connected to the motherboard circuit for fullduplex communication. When the device is running, the motherboard controls the serial port to send serial commands to the acquisition board, and the acquisition board transmits the original observation data to the motherboard according to different instructions., The mainboard directly stores the data and sends the data to the receiving server remotely when the remote communication task is started.

\subsection{Data Storage Module}

There may be communication interruption or data loss during remote communication [13]. In order to prevent the device from retaining the original data when there is a problem with the remote communication data transmission of the system, the device uses an external SD card to store the backup data.SD card and processor use SPI protocol to complete data storage and reading. The circuit is shown in Fig. 3. 


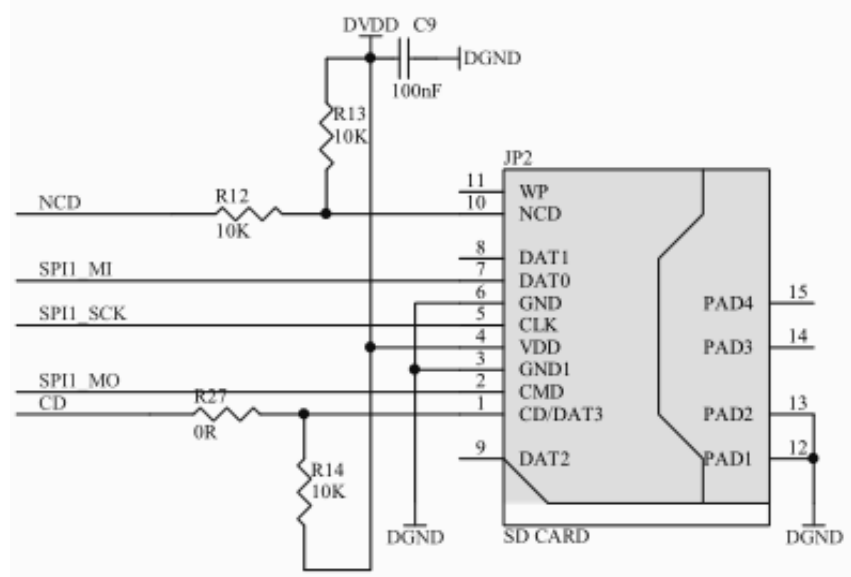

Fig. 3:SD card data storage

\subsection{Power Module}

In order to comprehensively and dynamically grasp the real-time changes of the concentration, water volume, and physical indicators of the water environment in the Yongding River Basin, the acquisition of energy in the field monitoring system is very important for the operation of its equipment [14]. In order to ensure that the equipment is unattended for many years, the automatic operation system uses the renewable energy sun as the energy source of the equipment. The entire solar intelligent power management system is shown in Fig. 4:

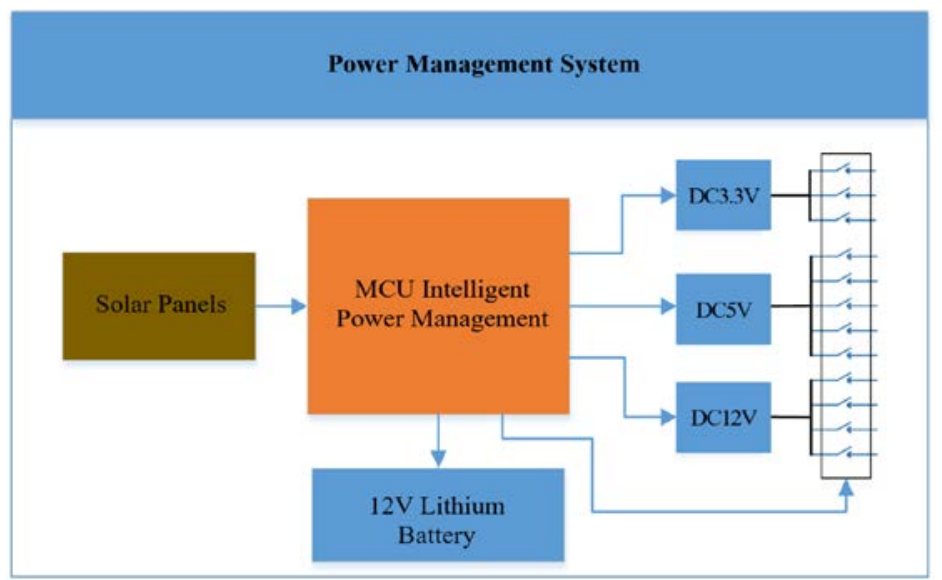

Fig. 4: Solar Intelligent Power Management System

The solar panel used has a maximum power of $60 \mathrm{~W}$ and a maximum output voltage of $18 \mathrm{~V}$. The charging voltage of the lithium battery is $12 \mathrm{~V}$, so in order to ensure the normal charging voltage for the lithium battery, the hardware circuit uses the LM5116 chip to realize the half-bridge switching power supply to perform DC-DC synchronous step-down control on the output voltage of the solar panel.

Low-temperature lithium-ion battery: 12V, 40Ah low-temperature lithium-ion battery provides energy input and output for the system. The battery can withstand low temperatures and theoretically has a capacity of $480 \mathrm{Wh}$ when fully charged. It supplements electricity through solar power to provide energy for the system.

Solar panels: convert solar energy into electricity and store it in batteries.

Debugging information output module: This module can output the operating information and energy status of the system through the RS232 interface, and can also realize the function of remote control to obtain the status information of all aspects of the equipment in real time.

Power output module: This module provides multi-channel voltage output (12V, 3.3V and 5V), each channel can be individually opened or closed under the control of the MCU, so the user can remotely control by sending command messages remotely Power supply. 


\subsection{Communication Module}

The equipment is designed to complete water and water collection and transmission under unattended conditions, so RS232 interface is reserved on the motherboard to complete remote communication tasks. The means of remote communication can be $4 \mathrm{G}$ and ZigBee communication, and can also be customized according to requirements. Through a data transmission module of RS232 to Ethernet interface, the USART protocol data is converted to UDP protocol data. Then the data is packaged into a short message through one of the communication modules and sent to the background data center through microwave communication. The communication of the system is duplex communication, so it can complete the remote setting, debugging and maintenance of the equipment.

\subsection{Unmanned Vessel (buoy) Design}

The system adopts unmanned ship carrying design, which can be moved and positioned by remote control and anchor automatically. The design and materials are shown in Figures 5.
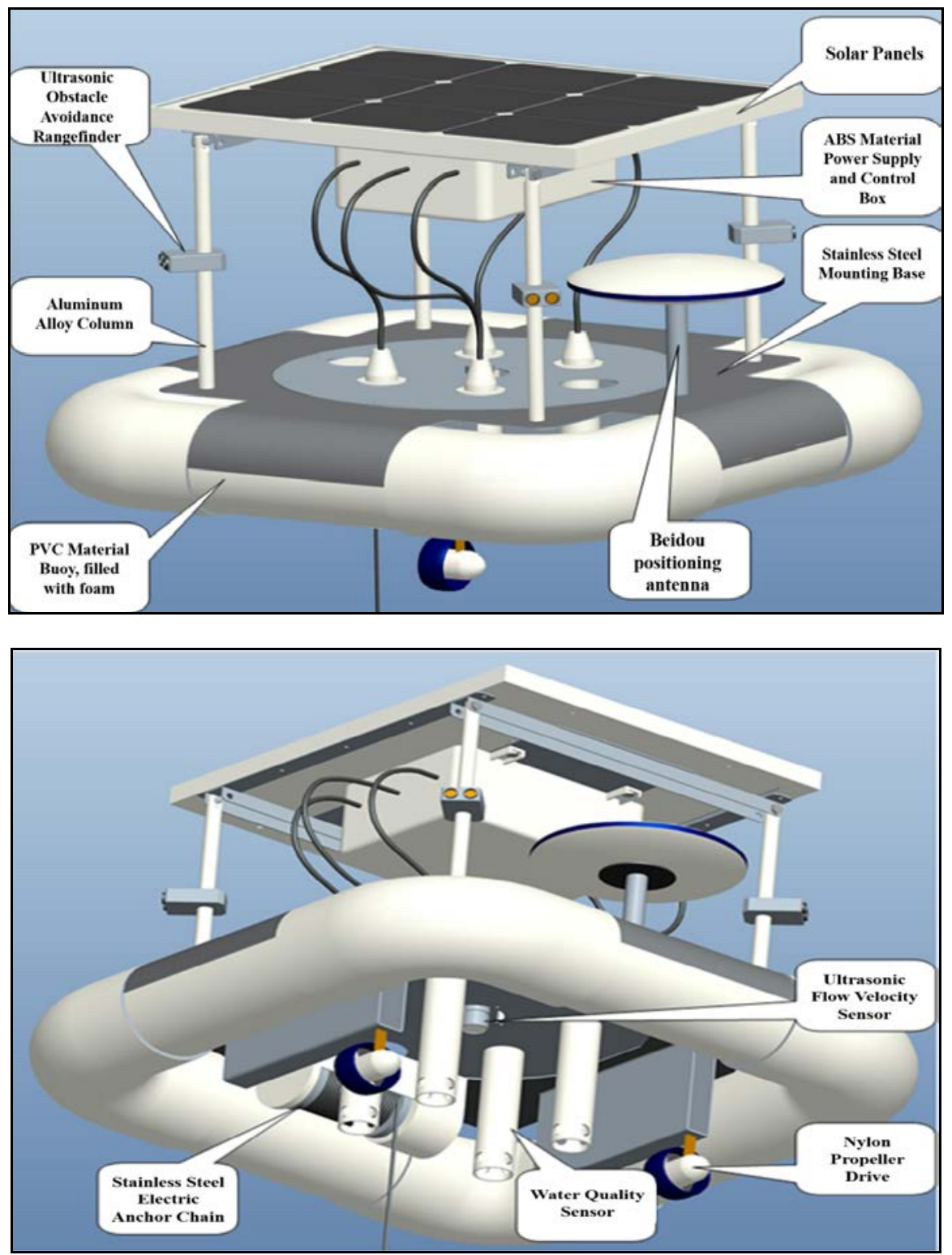

Fig. 5: Unmanned ship design 1 and Unmanned ship design 2

\subsection{Embedded Software System}

The software of this monitoring device is based on the self-developed embedded operating system, which has the advantages of fewer codes and more independence from hardware. The data acquisition, sending and receiving of the whole platform and the normal operation of each part of the function are controlled by this system [15]. The structure of the operating system is shown in Figure 6 . 


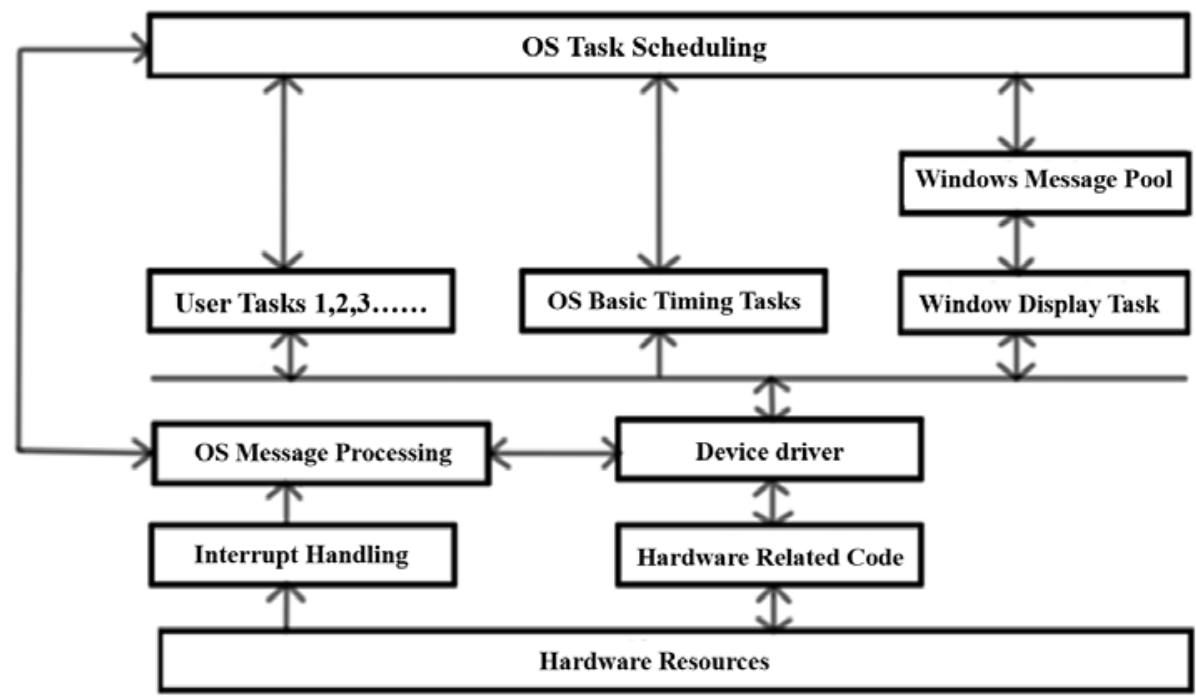

Fig. 6: System software structure diagram

The main functions of the embedded operating system are as follows: main task system module with standby function; timing management; data acquisition; wireless transmission; data storage; parameter setting and system calibration; remote wireless upgrade and real-time clock.

- System main task module (system with system standby function)

Before the system enters the standby state, it is necessary to determine the task to be performed after the system next wakes up, such as: only perform the sampling task;(2)open the communication module to upload the sampled data;(3)open the communication module and test whether the link with the server is normal;(4) open the GPS module for clock calibration and coordinate positioning.

In the system, there are several places to call the standby program or set the sleep flag: (1)GPS data collection is completed; (2) the data transmission module completes the transmission; (3) complete time calibration and coordinate positioning of GPS module; (4)the battery voltage is too low.

As shown in Fig 6, only after all of the following tasks are completed is the standby state allowed to enter (except for low voltage).

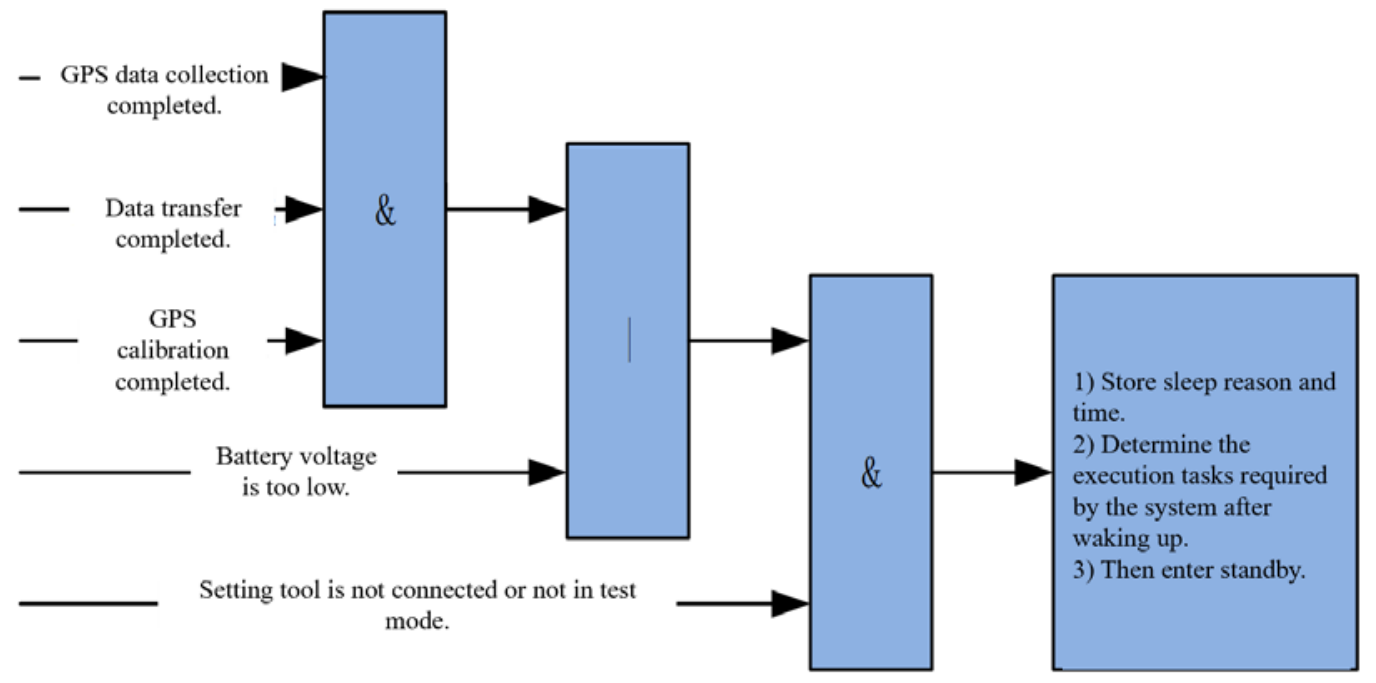

Fig. 7: Standby logic diagram of the system

- Timing module

Timing module is divided into two situations: (1) The module which has the function of sleep system needs to provide a semaphore to start sampling. The starting time of parameter acquisition and data transmission module had better be staggered in time in this kind of system; (2) If the system has been 
working, there is no need to standby, but the module needs to provide start sampling and data transmission module. In addition, sampling, data transmission, and GPS timing calibration should also be controlled through setup tools or remote networks.

\section{- Data collection}

Data collection is mainly to complete the sensor collection task and the observation data storage, as well as the solar voltage and battery voltage simulation data collection.

- Wireless transmission

The wireless data transmission module should be made into a standard send and receive function. The following functions are mainly completed through this module:(1) upload data collection information which is stored in an external SD card. When the data is uploaded, the signal strength and real-time battery voltage of the module should be uploaded so as to observe the status of the system;(2) make a remote wireless upgrade;(3) set system parameters and upload system status information in remote control; 4.all communications are checked and message confirmed.

- Data storage

The data storage in the system is roughly divided into three parts:(1) IAP remote upgrade program store: storing upgrade packages received by the wireless module;(2) sampling data: stored in bars;(3) system work log information: storing the information and state of the system when it is working, such as the time when the acquisition board is opened and the reason why the system enters the standby state.

- Parameter setting and system calibration

There are two ways to set parameters:(1) PC setup tool: system calibration and sampling data can be observed in this mode;(2) remote wireless settings: some simple parameters can be set and the system also needs to return the necessary parameter information and system state information.

This module can independently control the wireless transmission module and the acquisition board to open.

- Remote wireless upgrade

The remote upgrade part is made into a generic module and is only associated with the IAP package in order to ensure compatibility with the functionality of later projects.

- Real-time clock

The real-time clock needs to have the function of waking up the system with the interrupt of the RTC itself. STM32F107 itself has the RTC module which is an independent running timer. It has a series of continuously running counters so as to provide the function of clock calendar. The RTC core and clock configuration are stored in the processor's internal backup domain, which means that the RTC's time settings can be restored when the system is awake or reset on standby. Therefore, the RTC function of the processor is used to provide the system with time system and alarm clock wake-up function.

\section{Conclusion}

The seasonal variation of river water quantity in northern China is obvious, and the water quality is greatly affected by human activities. The social and economic development level and basin area of northern China are vast, so it is difficult to establish a large-scale watershed monitoring network with high spatial and temporal accuracy composed of fixed stations. Aiming at the current watershed ecological compensation pilot implementation of the realistic demand, this paper designs the ecological compensation monitoring system of water quality and quantity based on wireless sensor network. The system realizes the real-time monitoring, collection, transmission, display and storage of the multi-dimensional information such as the physical properties of pollutants and hydrological conditions, which can be used for the setting of ecological compensation assessment objectives and performance evaluation and promotes the transformation of ecological compensation in the watershed from a single assessment of water quality across boundary sections to an assessment standard focusing on both water quality and water quantity for the whole watershed and multiple points. It can effectively solve the problem of high cost, few points and low precision of the current monitoring system, and provide data support and technical support for the development of ecological compensation for water quality and quantity in the watershed. Besides, the system has the advantages of 
simple structure, low cost, easy installation and maintenance. It can be popularized with a little modification and has a good application prospect. It is inevitable that due to the limitations of the currently selectable sensors, the system does not carry parameters such as total nitrogen and total phosphorus. These parameters or more are also factoring that need to be considered in the study of water quality and ecological compensation. The expansion aspect still needs continuous follow-up.

\section{Acknowledgements}

This research has been aided in part by "Study on ecological compensation mechanism of water quality and quantity in Yongding River Basin" (2018ZX07111-002-006), the sub topic of "Demonstration study on integrated water quality management in Yongding River Basin” (2018ZX07111-002), which is supported by Major national science and technology projects of China.

\section{References}

[1] Blundy-Canto G, Box V, Quintero M, Cruz-Garcia G S,et al. The Different Dimensions of Livelihood Impacts of Payments for Environmental Services (PES) Schemes: A Systematic Review. Ecological Economics. Sci. Eng.2018. 149: p. 160-183.

[2] Sanchez-Azofeifa G A, Pfaff A, Robalino J A and Boomhower J P. Costa Rica's payment for environmental services program: Intention, implementation, and impact. Conservation Biology. Sci. Eng. 2007. 21(5): p. 11651173.

[3] Brooks, D.B., L. Thompson and L. El Fattal. Water demand management in the Middle East and North Africa: Observations from the IDRC forums and lessons for the future. Water International.Sci.Eng.2007. 32(2): p. 193204.

[4] Wang L. Watershed Eco-Compensation Mechanism and Policy study in China. Procedia Environmental Sciences. Sci. Eng. 2010. 2: p. 1290-1295.

[5] R Yang, Y Zhang, X Li, L Zhang, M Sun.Innovate the ecological compensation mechanism of the Yongding River Basin to help the coordinated development of Beijing, Tianjin and Hebei. Ecological Economy.Chi.2019. 35(12): p.134-138.

[6] Z Sun, J Lan, Y Wu and H Zhou. Management and Practice of Water Environment Monitoring Station Network in the Yangtze River Basin. Yangtze River. Chi. 2017. 48(S2): p 28-31.

[7] J Zhao, S Jiang and M Qian. Some thoughts on the management system of water resources monitoring station network at the provincial boundary of the Huaihe River Basin.Zhi Huai. Chi.2015(12): p 34-36.

[8] X Chen, J Cai and X Xing. Research on water quality automatic monitoring network planning of Nanjing Qinhuai River Basin. Resources Economization \& Environmental Protection. Chi. 2020(10): p 48-49.

[9] Meng, J., et al. Increasing perfluoroalkyl substances and ecological process from the Yongding Watershed to the Guanting Reservoir in the Olympic host cities, China. Environment International.Sci.Eng.2019. 133: p. 105224.

[10] Lu, S., et al., Forty years' channel change on the Yongding River, China: patterns and causes. International Journal of River Basin Management. Sci. Eng. 2016. 14(2): p. 183-193.

[11] He, W., et al. Ecological risk assessment and priority setting for typical toxic pollutants in the water from BeijingTianjin-Bohai area using Bayesian matbugs calculator (BMC). Ecological Indicators. Sci. Eng. 2014. 45: p. 209218.

[12] Li, F., et al. Trivariate Copula Based Evaluation Model of Water Accessibility. Water Resources Management. Sci. Eng. 2019. 33(9): p. 3211-3225.

[13] Li, X., et al. Design and Implementation of a Wireless Sensor Network-Based Remote Water-Level Monitoring System. Sensors. Sci. Eng. 2011. 11(2): p. 1706-1720.

[14] Ma, Y., et al. Development and Application of an Atmospheric Pollutant Monitoring System Based on LoRa-Part I: Design and Reliability Tests. Sensors. Sci. Eng. 2018. 18(389111).

[15] Li, X., et al. The Combination of Ground-Sensing Network and Satellite Remote Sensing in Huailai County. IEEE Sensors Journal.Sci.Eng.2016. 16(10): p. 3819-3826. 